\title{
L'éléphantiasis péno-scrotal. A propos d'un cas
}

\author{
Hamadi FAKHFAKH, Hafedh KETATA, Amin BOUASSIDA, Amir MALLEK, \\ Ali BAHLOUL, Mohamed Nabil MHIRI
}

Service d'Urologie du CHU Habib Bourguiba, Sfax, Tunisie

\section{RÉSUMÉ}

Le lymphoedème scrotal ou éléphantiasis scrotal est une pathologie rare en dehors des zones d'endémie filarienne. Nous rapportons un cas d'éléphantiasis scrotal idiopathique chez un patient âgé de 52 ans. Le traitement a consisté en une résection chirurgicale du tissu pathologique et une reconstruction scrotale, avec un bon résultat esthétique.

Mots clés : éléphantiasis, lymphœdème, scrotum, chirurgie

\section{INTRODUCTION}

L'éléphantiasis péno-scrotal se définit comme étant une augmentation du volume des bourses qui peut atteindre des tailles monstrueuses, ce qui va être responsable d'un aspect inesthétique, d'un impact sexologique du fait de l'enfouissement de la verge, et d'un préjudice psychologique.

Son diagnostic est clinique et sont traitement relève de la chirurgie. Cette pathologie est rare en dehors des zones d'endémie filarienne.

Nous rapportons un cas d'éléphantiasis scrotal dont l'origine est non filarienne.

\section{OBSERVATION}

II s'agit d'un homme âgé de 52 ans, Nord Africain, circoncis à son jeune âge, père de 5 enfants, qui consulte pour une augmentation progressive du volume des bourses et de la peau pénienne, évoluant depuis 4 ans, gênant l'activité sexuelle et sans troubles mictionnels associés.
Aucun antécédent de maladie vénérienne, ni d'infection streptococcique, ni de séjours en zone d'endémie filarienne, ni de traumatisme scrotal, ni de chirurgie ou de radiothérapie pelvienne n'a été noté.

L'examen clinique a mis en évidence un éléphantiasis péno-scrotal avec des bourses de $70 \mathrm{~cm}$ de diamètre et un enfouissement de la verge en doigt de gant avec une peau pénienne remaniée. La peau scrotale est épaissie et cartonnée. On a noté également la présence d'un lymphœdème du membre inférieur droit (Figure 1). Par ailleurs, le patient n'a pas de chylurie.

Un bilan étiologique, en particulier la recherche de microfilaires dans le sang et dans les urines, était négatif. La rectoscopie pratiquée a éliminé une étiologie infectieuse périanale associée. L'exploration des axes vasculaires des membres inférieurs par une écho-doppler n'a pas trouvé de stase ni d'obstruction. La tomodensitométrie abdominopélvienne a objectivé un épaississement des parois scrotales sans autres anomalies décelables.

Le diagnostic d'éléphantiasis scrotal primitif a été retenu et une exérèse avec plastie scrotale ont été effectuées.

Une incision inguinale bilatérale permettant de libérer les cordons spermatiques et les deux testicules a été pratiquée, puis celle-ci a été complétée par une incision circonférentielle sur le sillon balano-prépucial avec déshabillement de la verge permettant ainsi de repérer l'urètre spongieux et le suivre jusqu'au noyau fibreux central du périnée avec un accès à la face antérieure du rectum tout en respectant le sphincter anal. Ce qui a permis de faire une exérèse en bloc du tissu pathologique en ménageant deux languettes latérales. Celles-ci, avec la peau restante du prépuce et le tissu périnéal jugé sain ont été utilisées

\section{Correspondance :}

Dr Hamadi FAKHFAKH, Hôpital Habib Bourguiba, Service d'Urologie, 3029 Sfax, Tunisie - Tel +21698639784 Fax +21674243427 - Email h.fakhfakh@laposte.net 
dans la plastie du scrotum et le recouvrement de la verge. L'aspect final postopératoire est esthétiquement satisfaisant (Figure 2).

L'étude anatomopathologique était en faveur d'un lymphoedème scrotal d'origine inflammatoire. Les suites opératoires immédiates étaient simples. Récidive après un recul d'une année.

\section{DISCUSSION}

L'éléphantiasis péno-scrotal est causé par une infiltration anormale de liquide riche en protéines au niveau du tissu sous-cutané, qui serait due à une altération locale de la pression oncotique ou hydrostatique. Classiquement, il représente la manifestation dermatologique de l'obstruction mécanique des canaux lymphatiques par l'inflammation et la fibrose ou par des vers adultes de filaire [18].

La cause la plus fréquente du lymphœdème scrotal est la filariose lymphatique. Cette parasitose est observée essentiellement dans les pays tropicaux et sub-tropicaux [2], elle affecte surtout l'homme adulte de la quarantaine [12]. En dehors des zones d'endémie filarienne, l'éléphantiasis scrotal peut être congénital, secondaire ou plus souvent idiopathique.

Les causes acquises sont dues à une obstruction mécanique ou inflammatoire chronique pouvant êtres consécutives à une tumeur abdominale ou pelvienne, une chirurgie carcinologique ou une radiothérapie pelviennes $[3,7]$, une maladie de Kaposi, une infection génitale à streptocoque ou à chlamydia [17], une lymphogranulomatose vénérienne [14], ou à une hypoprotidémie [24].

Le lymphœdème scrotal congénital ou primitif est causé par une malformation du système lymphatique d'origine dysplasique non régressive s'intégrant dans le cadre des lymphangiomes [20, 24]. Dans notre cas la cause de l'éléphantiasis est restée indéterminée.

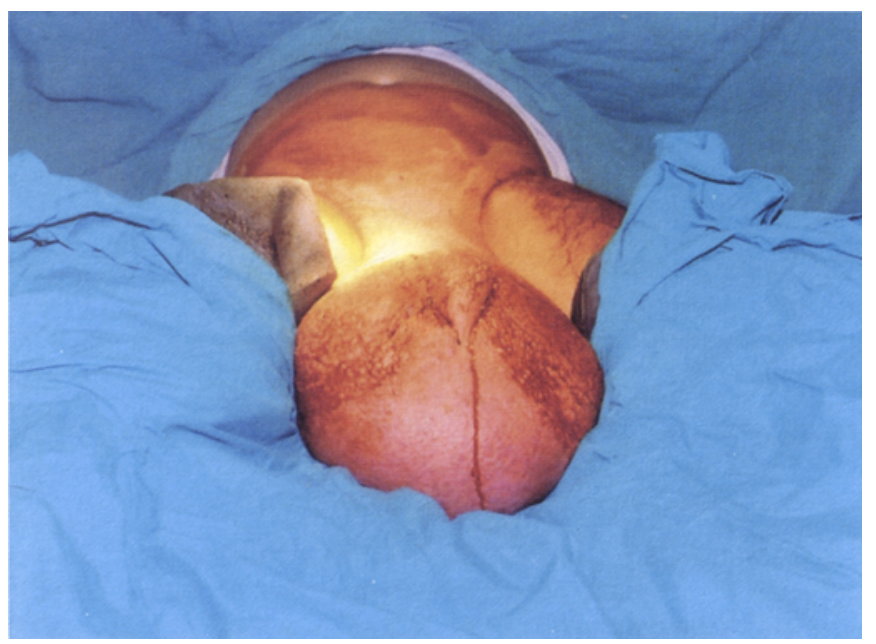

Figure 1 : aspect pré-opératoire de l'éléphantiasis penoscrotal avec enfouissement de la verge.
Scott McDougal a proposé récemment une classification de ces lymphœdèmes des organes génitaux externes en fonction du caractère congénital ou acquis, sporadique ou héréditaire, et de l'âge d'apparition [21].

Par ailleurs, la localisation pénienne isolée reste rare [2], elle est localisée à la peau pénienne et limitée par la couronne du gland [4]; l'atteinte est le plus souvent péno-scrotale épargnant en général le contenu épididymo-testiculaire [15]. Dans certains cas, la peau pénienne est épargnée et l'atteinte est scrotale pure [10].

Le diagnostic est clinique, il est évoqué devant une augmentation importante du volume de la bourse au dépend de la peau scrotale devenant épaisse, cartonnée et perdant de son élasticité $[8,25]$. L'atteinte pénienne concomitante peut entraîner un enfouissement de la verge en doigt de gant gênant les rapports sexuels et parfois la miction $[2,18]$.

En cas d'étiologie filarienne, l'échographie scrotale est une technique non invasive permettant de localiser $80 \%$ des vers adultes de Wuchereria Bancrofti chez les patients infectés en montrant une dilatation des vaisseaux lymphatiques et le mouvement des vers (danse filarienne) $[1,5,6$, 22].

L'échographie doppler permet de rechercher un obstacle sur les axes vasculaires des membres inférieurs ; en cas de filariose, elle peut mettre en évidence le mouvement des vers adultes au niveau des vaisseaux lymphatiques [6]. Elle permet aussi de surveiller l'évolution sous traitement antifilarien [11].

L'imagerie par résonance magnétique permettrait une caractérisation tissulaire bien corrélée avec les données anatomopathologiques, précisant la localisation du tissu sain de façon à pouvoir limiter la résection chirurgicale et faciliter la reconstruction scrotale [16, 23].

En dehors des éléphantiasis scrotaux survenant après une radiothérapie pour métastases ganglionnaires pelviennes

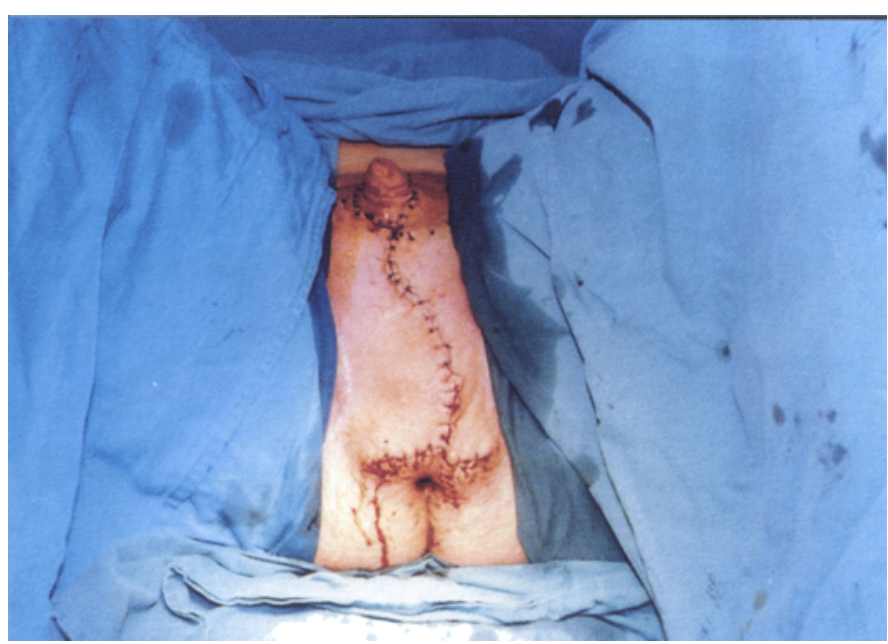

Figure 2 : aspect définitif post-opératoire après résection de la peau scrotale et plastie de la peau pénienne. 
massives, où un traitement conservateur est préconisé par l'espérance de vie très limitée, le traitement repose sur la chirurgie. II sera entrepris après antibiothérapie adaptée et stabilisation du processus inflammatoire $[4,16]$. Une exérèse large de la paroi scrotale pathologique réalisant en même temps une lymphangiectomie superficielle totale sera entreprise; il est recommandé de commencer par une libération des cordons spermatiques et des testicules par une incision à hauteur de l'orifice inguinal superficiel [2].

L'exérèse chirurgicale radicale est suivie d'une plastie scrotale pouvant faire appel à plusieurs procédés :

- Deux lambeaux scrotaux bilatéraux peuvent êtres préservés permettant la reconstruction d'un néo-scrotum. II s'agit d'une technique utilisée par la plupart des auteurs et qui donne de bons résultats $[12,13,18]$.

- Lambeaux cutanés pédiculés inguinaux ou supra pubiens $[2,3,16]$.

- L'utilisation de la peau libre mince a été décrite [18, 25], mais cette dernière pourrait induire des troubles de la spermatogenèse par modification de la régulation thermique testiculaire.

Pour la reconstruction de la peau pénienne, on peut recourir à la greffe de peau libre mince disposée de façon spiralée afin d'éviter une rétraction sur le pénis [3, 4, 9, 13, 19]. Parfois, l'importance de la stase lymphatique chronique peut atteindre le cordon spermatique et les testicules, nécessitant alors une orchidectomie bilatérale [14].

\section{CONCLUSION}

L'éléphantiasis scrotal est une pathologie rare. Une enquête étiologique est nécessaire avant d'entamer le traitement. L'exérèse chirurgicale avec plastie scrotale donne un résultat fonctionnel et esthétique excellent permettant aux patients de reprendre vie normale.

\section{REFERENCES}

1. AMARAL F., DREYER G., FIGUEREDO-SILVA J. et al. : Live adult worms detected by ultrasonography in human Bancroftian filariasis. Am. J. Trop. Med. Hyg., 1994, 50 : 753757.

2. ANDAPAT M.C., MOHAPATRO S.K., PATRO S.K. : Elephantiasis of the penis and scrotum. A review of 350 cases. Am. J. Surg., 1985, $149:$ 686-690.

3. APEROS J., ANIGIAN G. : Reconstruction of penile and scrotal lymphedema. Ann. Plast. Surg., 1991, 27 : 470-472.

4. DEVRIES C.R. : The role of the urologist in the treatment and elimination of lymphatic filariasis worldwide $\mathrm{Br}$. J. Urol., 2002, 89 : s37-s43.

5. DREYER G., SANTOS A., NOROES J., ADDISS D. : Proposed panel of diagnostic criteria, including the use of ultrasound, to refine the concept of endemic normals in lymphatic filariasis. Trop. Med. Int. Health, 1999, 4 : 575-579.

6. FARIS R., HUSSAIN O., EL SETOUHY M., RAMZY R.M., WEIL G.J. : Bancroftian filariasis in Egypt : visualization of adult worms and subclinical lymphatic pathology by scrotal ultrasound. Am. J. Trop. Med. Hyg., 1998, $59: 864-867$.

7. FRANCA A.A., JUNIOR A.N., CUNRATH G.S., RONCHI L.S., NETINHO J.G. : Elefantiase escrotal pos cirurgia e radioterapia. HB Cientifica. 2002, $9: 84-87$.

8. FULMER L.R., FYE C. : Elephantiasis of the scrotum. J. Clin. Ultr., 1982, $10: 143-144$.

9. HOLMAN C.M. J.R., ARNOLD P.G., JURKIEWICZ M.J., WALTON K.N. : Reconstruction of male external genitalia with elephantiasis. Urology, 1977, $10: 476-478$.

10. HORNBERGER B.J., ELMORE J.M., CLAUS G. ROEHRBORN C.G. : Idiopathic scrotal elephantiasis. Urology, 2005, $65: 389$.

11. HUSSEIN O., SETOUHY M.E., AHMED E.S. et al. : Duplex Doppler sonographic assessment of the effects of diethylcarbamazepine and albendazole therapy on adult filarial worms and adjacent host tissues in bancroftian filariasis Am. J. Trop. Med. Hyg., 2004, 71 : 471-477.

12. KHEDROV L.G. : Reduction perineoplasty for scrotal elephantiasis : a method of surgical reconstruction of the penis and scrotum. J. Urol.,1976, $116: 679-680$

13. KONETY B.R., COOPER T., FLOOD H.D., FUTRELL J.W. : Scrotal elephantiasis associated with hidradenitis suppurativa. Plast. Recons. Surg., 1996, 97 : 1243-1245.

14. KUEPPER D. : Giant scrotal elephantiasis. Urology, 2005, 65 : 389.

15. MIANNE D., JACOB F. : Pathologie inflammatoire du scrotum. Enc. Med. Chir. Nephrol. Urol., 1999, 18-640-A-10-6.

16. NDOYE A., SYLLA C., GUEYE M.B.A. S.M., DIAGNE B.A. : Point of technique : Management of penile and scrotal elephantiasis. Br. J. Urol. Int., 1999,84 : 362-364.

17. NELSON R.A., ALBERTS G.L., KING LE J.R. : Penile and scrotal elephantiasis caused by indolent Chlamydia trachomatis infection. Urology, 2003, 61: 224.

18. OLLAPALLIL J.J., WATTERS D.A.K. : Surgical management of elephantiasis of male genitalia. Br. J. Urol., 1995, $76: 213-215$.

19. RAGHAVAIAH N.V. : Reconstruction of scrotal and penile skin in elephantiasis. J. Urol., 1977, $118: 541-546$.

20. ROSS J.H., KAY R., YETMAN R.J., ANGERMEIER K. : Primary lymphedema of the genitalia in children and adolescents. J. Urol., 1998, $160: 1485-1489$.

21. SCOTT MCDOUGAL $W$. : Lymphedema of the external genitalia. J. Urol., 2003, $170: 711-716$.

22. SHENOY R.K., JOHN A., HAMEED S., SUMA T.K., KUMARASWAMI V. : Apparent failure of ultrasonography to detect adult worms of Brugia malayi. Ann. Trop. Med. Parasitol., 2000, $94: 77-82$.

23. SLAMA A., JAIDANE M., ELLEUCH A., BEN SORBA N., YACOUBI M.T., MOSBAH A.F. : Traitement chirurgical d'un éléphantiasis péno-scrotal. Prog. Urol., 2002, 12 : 337-339.

24. TAMMER M.E., PLOGMEIER K., SCHNEIDER W. : Surgical therapy of scrotal oedema in elephantiasis congenita hereditaria (Meige type). Urologe A. 2002, $41: 493-495$.

25. WILLE S., NIESEL T., BREUL J., HARTUNG R. : Elephantiasis of the legs with lichen sclerosis and atrophicus of the penis and scrotum. J. Urol., 1997, $157: 2262$. 


\author{
ABSTRACT \\ Penoscrotal elephantiasis : a case report
}

Hamadi FAKHFAKH, Hafedh KETATA, Amin BOUASSIDA, Amir MALLEK, Ali BAHLOUL, Mohamed Nabil MHIRI

Massive scrotal lymphoedema with gross genital deformation is called penoscrotal elephantiasis. It is a rare syndrome outside of filariasis endemic regions. It is usually idiopathic and rarely congenital or secondary. It is both physically disabling and emotionally distressing for the patient. Surgical treatment is often necessary. The authors report a case of a patient with penoscrotal elephantiasis in whom no aetiology could be found. Complete surgical resection of the diseased tissue and scrotum and penile reconstruction were performed with good cosmetic and functional results.

Key words: elephantiasis, lymphoedema, scrotum, surgery 